\title{
Phosphorylation and nuclear translocation of STAT3 regulated by the Epstein-Barr virus latent membrane protein 1 in nasopharyngeal carcinoma
}

\author{
YI-PING LIU, YUN-NIAN TAN, ZHEN-LIAN WANG, LIANG ZENG, \\ ZHONG-XIN LU, LI-LI LI, WEI LUO, MIN TANG and YA CAO \\ Cancer Research Institute, Xiangya School of Medicine, Central South University, \\ 110 Xiangya Road, Changsha, Hunan 410078, P.R. China
}

Received July 17, 2007; Accepted August 27, 2007

\begin{abstract}
The Epstein-Barr virus (EBV) latent membrane protein 1 (LMP1) oncoprotein has been shown to mediate activation of the signal transducer and activator of transcription 3 (STAT3). In the present study, we delineated the mechanism by which LMP1 stimulates STAT3 in a human nasopharyngeal carcinoma (NPC) cell line. LMP1 stimulated STAT3 Tyr 705-dependent nuclear accumulation, as well as the phosphorylation of STAT3 at both Tyr 705 and Ser 727. Treatment of cells with interleukin-6 neutralizing antibody inhibited the phosphorylation of STAT3 Tyr 705 and Ser 727. The differential phosphorylation of STAT3 was found to be a result of activation of Janus kinase 3 (JAK3) and extracellular signal-regulated kinase (ERK). The biological significance of JAK3-mediated activation of STAT3 Tyr 705 phosphorylation was further assessed by treating the cells with an inhibitor (WHI-P131) of JAK3. Inhibition of ERK activity by an inhibitor (PD98059) of MAPK/extracellular signal-regulated kinase kinase (MEK1) decreased the LMP1-induced activation of STAT3 Ser 727. Furthermore, immunohistochemical analysis showed an increased nuclear STAT3 Tyr 705 staining in LMP1-positive cells and STAT3 Tyr 705 phosphorylation related to NPC stages III and IV. Demonstration of the involvement of different kinases in LMP1-induced STAT3 activation supports the involvement of the JAK/STAT and mitogen-activated protein kinase (MAPK)/ERK signaling pathways in the regulation of STAT3 activation by LMP1.
\end{abstract}

\section{Introduction}

The Epstein-Barr virus (EBV), a $\gamma$ herpes virus, is associated with many malignancies, including Burkitt's lymphoma, nasopharyngeal carcinoma (NPC), and Hodgkin's disease (1).

Correspondence to: Dr Ya Cao, Cancer Research Institute, Xiangya School of Medicine, Central South University, 110 Xiangya Road, Changsha, Hunan 410078, P.R. China

E-mail:ycao98@public.cs.hn.cn

Key words: Epstein-Barr virus, latent membrane protein 1, nasopharyngeal carcinoma, JAK/STAT, MAPK/ERK
A subset of genes, including latent membrane protein 1 (LMP1), is expressed when EBV transforms resting B lymphocytes into lymphoblastoid cell lines (LCLs). Among the products expressed by EBV during latent infection, LMP1 is regarded as the principal oncoprotein of EBV $(2,3)$.

LMP1 is a $63-\mathrm{kDa}$ integral membrane protein consisting of a short highly charged cytoplasmic amino terminus of 23 amino acids; 6 hydrophobic transmembrane domains required for protein aggregation, and a long acidic cytoplasmic carboxyl terminus of 200 amino acids $(4,5)$. The carboxyl terminus comprises two functional domains: the membraneproximal C-terminal activation region-1 (CTAR1) and the membrane-distal CTAR2 $(6,7)$. LMP1 can mediate the activation of NF- $\mathrm{\kappa B}$ through CTAR1 as well as the activation of both NF- $\mathrm{B}$ and AP-1 through CTAR2. It has been reported that there exists a new domain between CTAR1 and CTAR2 named CTAR3 that is associated with the Janus kinase/signal transducer and activator of transcription (JAK/STAT) signaling pathway (8).

Mitogen-activated protein (MAP) kinases represent a family of serine/threonine protein kinases comprising extracellular signal-regulated kinase (ERK)1/ERK2, p38/HoG1 (p38), and c-Jun N-terminal kinase/stress-activated protein kinase (JNK/SAPK) (9-11). Our previous work shows that LMP1 can activate the AP-1 family by activating JNK and regulating the transcription of downstream genes. JNK plays a vital role in the LMP1 signaling pathways. LMP1 promotes JNK phosphorylation by CTAR2 mainly through TRAF/ TRADD compounds and hence activates AP-1 (12). In addition, LMP1 can activate the Ras signaling pathway via the Ras/MAPKKK/MAPKK/ERK/Ets-1 signal (13).

STAT3 is a key molecule through which receptors of multiple cytokines and growth factors perform their functions. It plays a significant role in the promotion of cell proliferation $(14,15)$, the inhibition of cell apoptosis (16), immune escape $(17,18)$, angiogenesis (19), and the invasion and metastasis of tumor cells (20-22). STAT3 is thought to play a role in carcinogenesis through its abnormal signal transduction. Phosphorylation of tyrosine 705 (Tyr 705) and serine 727 (Ser 727) are the important functional forms of STAT3. After its activation with the phosphorylation of Tyr 705, STAT3 forms dimers with itself or with STAT1, translocates to nuclei, and then binds to specific DNA sequences and starts 
the transcription of multiple related downstream genes. It has been previously reported that LMP1 can promote STAT3 Tyr 705 phosphorylation and enhance its DNA binding activity in B lymphocytes $(8,23)$. STAT3 Ser 727 phosphorylation is required for the maximal activation of STAT3 signaling, and Tyr 705 phosphorylation alone is not sufficient for the obligatory role of STAT3 signaling in oncogenesis (24-26).

LMP1 expression can instigate an interleukin (IL)-6mediated signaling loop that renders the cell independent of the initial stimulus and provides continuous STAT3 Tyr 705 activation (27). In B cells and 293 cells, it has been reported that LMP1 can activate the JAK/STAT signaling pathway via CTAR3 (8). Our previous research showed that, in NPC cells, LMP1 can activate the JAK/STAT signaling pathway with the cooperation of the CTAR2 and CTAR3 domains, and that vascular endothelial growth factor (VEGF) can be upregulated by LMP1 through the activation of STAT3 $(28,29)$.

In the present study, we investigated the molecular mechanisms underlying STAT3 phosphorylation induced by LMP1, specifically addressing the role of ERK1/2 and the JAK3 signaling pathway in mediating LMP1-induced translocation of STAT3 to the nucleus. Our finding indicates that LMP1 protein expression has a significant correlation with the prognosis of NPC which may be associated with the activation of STAT3 phosphorylation.

\section{Materials and methods}

Cell lines and culture. CNE1 is an LMP1-negative, highly differentiated NPC cell line. CNE1-LMP1 is a stably transfected cell line, established by introducing LMP1 cDNA into the CNE1 cell, in which LMP1 is highly expressed. These cells were maintained in RPMI-1640 (Gibco BRL) medium supplemented with $10 \%$ heat-inactivated fetal calf serum. Tet-on-LMP1-HNE2 is an established cell line using a newly developed Tet-on gene expression system in which LMP1 is highly expressed in a dose-dependent manner. Tet-on-LMP1HNE2 cells were cultured in RPMI-1640 medium with $100 \mathrm{mg} / \mathrm{l}$ of G418 and $50 \mathrm{mg} / \mathrm{l}$ of hygromycin supplemented with $10 \%$ fetal calf serum. To induce LMP1 expression, Teton-LMP1-HNE2 cells were treated with doxycycline at a dosage of $0.6 \mu \mathrm{g} / \mathrm{ml}$ (30). All cells were grown at $37^{\circ} \mathrm{C}$ in a humidified atmosphere of $5 \% \mathrm{CO}_{2}$ and $95 \%$ air.

Reagents. The MAPK/extracellular signal-regulated kinase kinase (MEK1) inhibitor, PD98059, purchased from Cell Signaling Technology, was dissolved in dimethyl sulfoxide and stored at $-20^{\circ} \mathrm{C}$. WHI-P131, a JAK3 inhibitor, was kindly provided by Dr Jiming Wang (National Institutes of Health, Bethesda, MD, USA). The BCA Protein Assay Reagent Kit, Supersignal Chemiluminescence System (ECL), and NE-PER Nuclear and Cytoplasmic Extraction Reagents Kit were from Pierce Chemical (Rockford, IL, USA). The p44/p42 MAP Kinase Assay Kit was purchased from Cell Signaling Technology.

Western blot analysis. To induce LMP1 expression, Tet-onLMP1-HNE2 cells were treated with doxycycline (Dox) $0.6 \mu \mathrm{g} / \mathrm{ml}$ for $24 \mathrm{~h}$ before harvesting. To neutralize IL-6, anti-human IL-6 neutralizing antibody (R\&D Systems) was added to the medium at 10 or $20 \mu \mathrm{g} / \mathrm{ml}$ for $24 \mathrm{~h}$ before harvesting. Cells were treated with different kinase inhibitors for $2 \mathrm{~h}$ before harvesting. Nuclear extracts were prepared using the NE-PER Nuclear and Cytoplasmic Extraction Reagents Kit. Protein concentrations were determined using the BCA Protein Assay Reagent Kit, with bovine serum albumin as a standard.

Whole cell lysates were prepared by sonicating cell pellets in sample buffer and boiling for 5 min before loading onto the gel. Proteins were fractionated on $8 \%$ polyacrylamide gels and blotted onto a nitrocellulose membrane (Bio-Rad), following the manufacturer's protocol. Antibodies for detecting phosphorylated STAT were purchased from Cell Signaling Technology and used at a 1:1000 dilution in phosphatebuffered saline (PBS) with 5\% skim milk. The LMP1 antibody was obtained from Dako. Horseradish peroxidaseconjugated secondary antibody and the ECL Color Developing Kit were purchased from Pharmacia-Amersham and used according to the manufacturer's instructions. The antibodies used were as follows: LMP1 (cs1, Dako), STAT3 (\#9132, Cell Signaling Technology), phospho-Stat3 (705) (\#9136, Cell Signaling Technology), phospho-Stat3 (Ser 727) (\#9134, Cell Signaling Technology), actin (sc-8432), nucleolin (MS-3) (SC-8031), anti-human IL-6 neutralizing antibody (AB-206NA, R\&D), p-ERK (E-4) (sc-7383, Santa Cruz), and ERK (c-16) (93, Santa Cruz).

Immunofluorescence analysis. Cellular localization of proteins was investigated by immunofluorescence. Cells were washed with cold PBS and fixed with cold $3.7 \%$ polyformaldehyde for $30 \mathrm{~min}$. The primary antibody was diluted 1:200 with PBS and incubated with the cells at $4{ }^{\circ} \mathrm{C}$ overnight. Fluoresceinlabeled immunoglobulin $\mathrm{G}(\mathrm{IgG})$ was diluted 1:1000 with PBS and incubated with the cells to bind with the primary antibody. Cellular localization of proteins was observed using fluorescence microscopy or laser scanning confocal microscopy.

Flow cytometry. Cells were collected, rinsed with PBS, and suspended in $75 \%$ ethanol at $-20^{\circ} \mathrm{C}$ overnight. Fixed cells were centrifuged and washed with PBS twice, treated with $0.25 \%$ Triton X-100 for $5 \mathrm{~min}$, washed with PBS, incubated with the primary antibodies at $4{ }^{\circ} \mathrm{C}$ overnight, washed with PBS, and incubated with the diluted fluorescein isothiocyanate (FITC)-labeled anti-mouse IgG specific for pSTAT3 at room temperature for $30 \mathrm{~min}$. To detect the fluorescent intensity of certain proteins, cells were counterstained in the dark with $50 \mu \mathrm{g} / \mathrm{ml}$ phosphatidyl inositol (PI) and $0.1 \%$ ribonuclease A (RNase A) in $400 \mu 1 \mathrm{PBS}$ at $25^{\circ} \mathrm{C}$ for $30 \mathrm{~min}$. Stained cells were assayed and quantified using a FACSort flow cytometer (Becton Dickinson).

Immune complex kinase assay. ERK1/ERK2 activity was measured using a nonradioactive p44/42 MAP Kinase Assay Kit in accordance with the manufacturer's instructions. Briefly, cell lysates $(200 \mu \mathrm{g})$ were incubated with an antimonoclonal phospho-p44/42 MAP kinase (Thr 202 and Tyr 204) to selectively immunoprecipitate active ERK1/ERK2. Kinase assays were performed by incubating immunoprecipitates in $50 \mu \mathrm{l}$ of kinase buffer containing $200 \mu \mathrm{M}$ adenosine triphosphate and $20 \mu \mathrm{g}$ of Elk-1 fusion protein for 

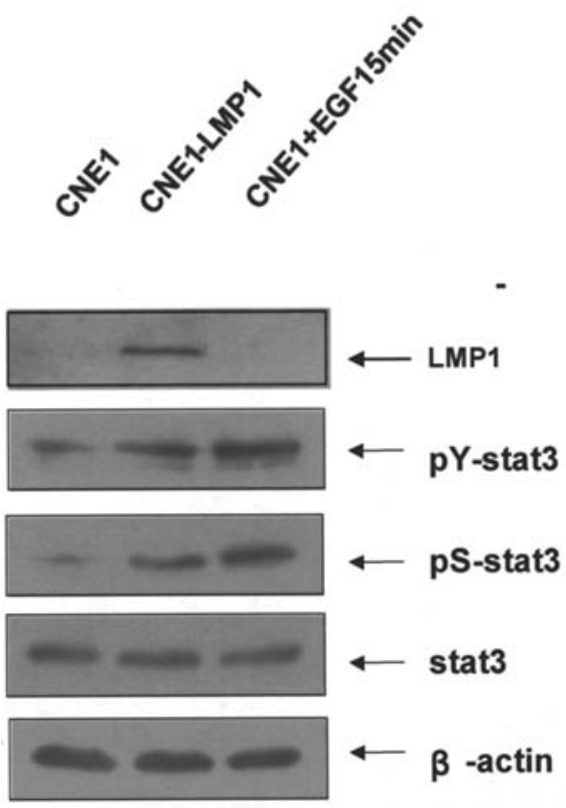

Figure 1. Effect of LMP1 on the phospho-STAT3 level in CNE1 and CNE1LMP1 cells. Cell lysates were prepared as described. Equal amounts of protein were separated by $10 \%$ sodium dodecyl sulfate-polyacrylamide gel electrophoresis (SDS-PAGE) and blotted onto a nitrocellulose membrane. Quantification of immunoband intensities was determined by densitometry scanning, with B-actin as an internal control. Lane 1, CNE1; lane 2, CNE1LMP1; lane 3, CNE1 treated with epidermal growth factor (EGF) (100 ng/ $\mathrm{ml}$ ) for $15 \mathrm{~min}$. LMP1 promoted STAT3 tyrosine 705 and serine 727 phosphorylation.

$30 \mathrm{~min}$ at room temperature. The reaction was terminated by the addition of $30 \mu 1$ of $2 \mathrm{X}$ Laemmli sample buffer, and samples were then boiled for $5 \mathrm{~min}$. The phosphorylation of Elk-1 was measured by Western blotting with an antiphospho-Elk-1 antibody (Ser 383).

Clinical samples and staging. A total of 40 NPC tissue samples were collected from the archives of the Department of Pathology at Hunan Province Cancer Hospital. Prior patient consent and approval from the Institute's Research Ethics Committee were obtained for the use of these clinical materials for research purposes. The disease stages of all patients were classified or reclassified according to the 1992 NPC staging system of P.R. China.

Double stain visualization. Immunostaining was performed by the A streptavidin-biotin immunoperoxidase complex method using a commercial kit (Dako).

Nonspecific reaction was blocked with normal mouse serum. The slides on which the cell lines grew were incubated with acetate-formaldehyde fixative for $20 \mathrm{~min}$ at room temperature and then washed in PBS, followed by quenching of endogenous peroxidase activity with $0.15 \%$ hydrogen peroxide in methanol. Next the slides were incubated with the primary antibodies (diluted $1: 100$ ) at $4^{\circ} \mathrm{C}$ overnight. After washing with $0.01 \mathrm{M}$ PBS, the slides were incubated with a biotinylated secondary antibody for $30 \mathrm{~min}$ and finally incubated with streptavidin peroxidase for $30 \mathrm{~min} .3,3^{\prime}-$ diaminobenzidine tetrahydrochloride $(0.05 \%)$ was used as the final chromogen. Counterstaining of the nucleus was achieved with hematoxylin.
Table I. The intensity of fluorescence of p-STAT3 and STAT3 in NPC cells.

\begin{tabular}{lccc}
\hline & $\begin{array}{r}\text { STAT3 } \\
\text { Tyr 705 }\end{array}$ & $\begin{array}{l}\text { STAT3 } \\
\text { Ser 727 }\end{array}$ & STAT3 \\
\hline CNE1 & 18.16 & 12.33 & 34.16 \\
CNE1-LMP1 & 27.82 & 21.29 & 35.46 \\
Tet-on-LMP1-HNE2 Dox 0 & 18.58 & 12.72 & 50.92 \\
Tet-on-LMP1-HNE2 Dox 0.6 & 29.69 & 20.62 & 55.66 \\
\hline
\end{tabular}

The intensity of fluorescence showed the effect of LMP1 on the phospho-STAT3 level and on the total STAT3 level in CNE1, CNE1-LMP1, Tet-on-LMP1-HNE2 Dox $0 \mu \mathrm{g} / \mathrm{ml}$, and Tet-onLMP1-HNE2 Dox $0.6 \mu \mathrm{g} / \mathrm{ml}$ cells as detected by flow cytometry. Control, without primary antibody

Statistical analysis. The significance of differences between experimental conditions was determined using the Spearman analysis and $\chi^{2}$ test.

\section{Results}

EBV-LMP1 upregulation of STAT3 phosphorylation in NPC. To define the regulation of LMP1 on STAT3 phosphorylation, we used CNE1 (LMP1-negative) and CNE1LMP1 as the cell models for the detection of STAT3 phosphorylation by Western blotting. CNE1 treated with $100 \mathrm{ng} /$ $\mathrm{ml}$ epidermal growth factor (EGF) was used as the positive control. CNE1-LMP1 had markedly higher STAT3 Tyr 705 (pY-Stat3) and Ser 727 (pS-stat3) phosphorylation than did CNE1, but STAT3 expression levels showed no distinct difference (Fig. 1).

To confirm this observation, fluorescent intensity analysis was performed using flow cytometry. We found CNE1-LMP1 had higher fluorescent intensity for both STAT3 Tyr 705 phosphorylation and STAT3 Ser 727 phosphorylation than did CNE1. Similarly, STAT3 Tyr 705 and Ser 727 phosphorylation was elevated by $0.6 \mu \mathrm{g} / \mathrm{ml}$ doxycycline in Teton-LMP1-HNE2 cells, in which the expression of LMP1 would be stimulated by doxycycline in a dose-dependent manner and LMP1 maximally induced by $0.6 \mu \mathrm{g} / \mathrm{ml}$ doxycycline (30). The data demonstrated that LMP1 had a marked effect in promoting STAT3 Tyr 705 and Ser 727 phosphorylation in the NPC cell lines (Fig. 2, Table I).

Previous studies support a positive feedback loop of IL-6induced STAT activation by LMP1 (27), and we were able to confirm these results using Tet-on-LMP1-HNE2 cells. Expression of LMP1 led to a striking increase in both Tyr 705 and Ser 727 phosphorylation of STAT3 protein (Fig. 3). By blocking IL-6 activation through the use of an anti-IL-6 neutralizing antibody, we found that activation of STAT3 in response to IL-6 was dependent on phosphorylation of both tyrosine and serine amino acid residues. The suppression of STAT3 Ser 727 phosphorylation was observed at $10 \mu \mathrm{g} / \mathrm{ml}$ during IL-6 inhibition, whereas the suppression of STAT3 Tyr 705 phosphorylation was observed at $20 \mu \mathrm{g} / \mathrm{ml}$. However, in doxycycline-untreated cells, IL-6 neutralizing antibody did 


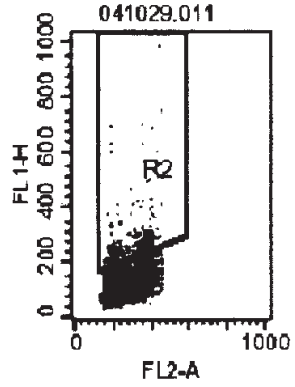

Tet-on-LMP1-HNE2 Dox0 $\mu \mathrm{g} / \mathrm{ml}$ Control

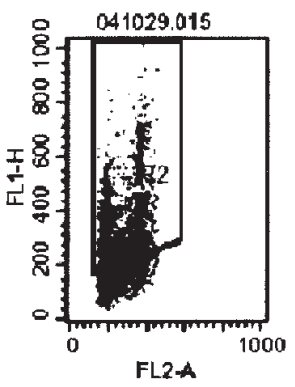

Tet-0n-LMP1-HNE2 Dox0 $\mu \mathrm{g} / \mathrm{ml}$ STAT3

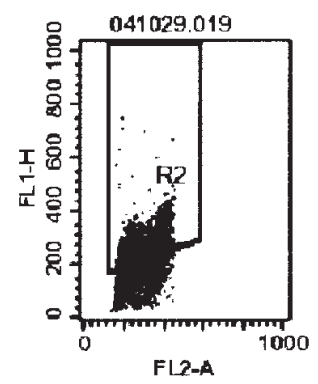

Tet-on-LMP1-HNE2 Dox0.6 $\mu \mathrm{g} / \mathrm{ml} \quad$ pY-STAT3

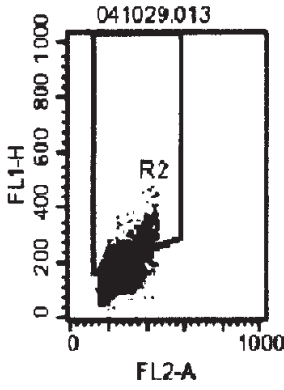

Tet-on-LMP1-HNE2 Dox0 $\mu \mathrm{g} / \mathrm{ml} \quad \mathrm{pS}-\mathrm{STAT3}$

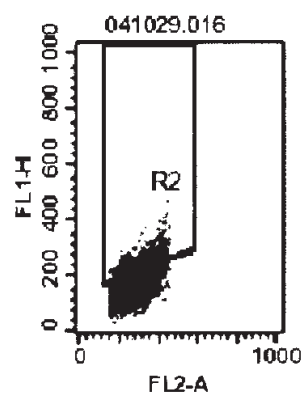

Tet-on-LMP1-HNE2 Dox0.6 $\mu \mathrm{g} / \mathrm{ml}$ Control

Tet-on-LMP1-HNE2 Dox0.6 $\mu \mathrm{g} / \mathrm{ml} \quad$ pS-STAT3

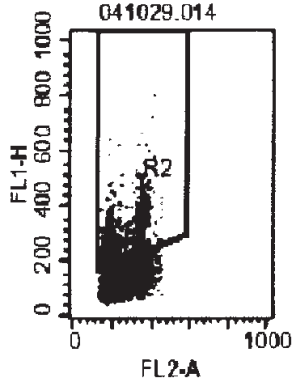

Tet-on-LMP1-HNE2 Dox0 $\mu \mathrm{g} / \mathrm{ml}$ pY-STAT3

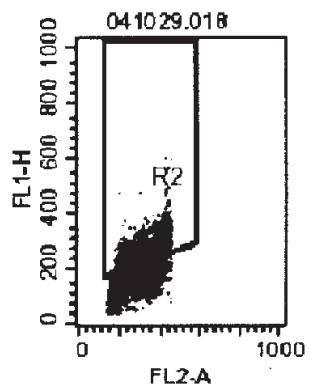




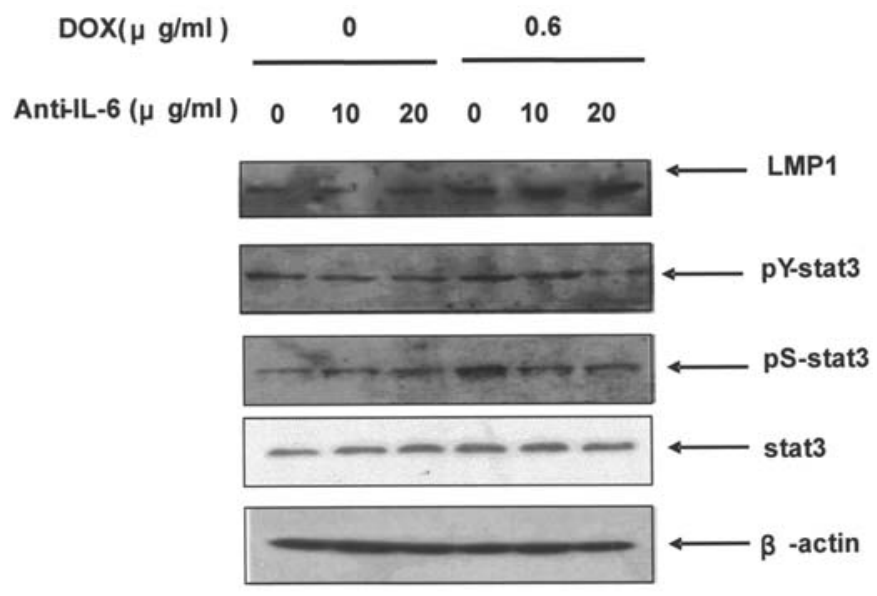

Figure 3. Anti-IL-6 neutralizing antibody prevents the ability of LMP1 to induce phosphorylation of STAT3 Tyr 705 and STAT3 Ser 727 in Tet-onLMP1-HNE2 cells. Tet-on-LMP1-HNE2 cells were stimulated with Dox $0.6 \mu \mathrm{g} / \mathrm{ml}$ and anti-IL-6 neutralizing antibody for $24 \mathrm{~h}$ before harvesting the lysates. Dox $0.6 \mu \mathrm{g} / \mathrm{ml}$ caused an increase in STAT3 Tyr 705 and STAT3 Ser 727 phosphorylation. However, the presence of the anti-IL-6 neutralizing antibody prevented the LMP1-induced phosphorylation of STAT3 Tyr 705 and STAT3 Ser 727 in a dose-dependent manner. Total cellular levels of STAT3 were used as loading controls

phosphorylated STAT3 (pS-stat3) in comparison to CNE1 cells (Fig. 4). Meanwhile, immunofluorescence analysis was performed to localize phosphorylated STAT3 (Tyr 705, Ser 727) in these cell lines. We found that CNE1-LMP1 had a relatively higher intensity of fluorescence of phosphorylated STAT3 (Tyr 705, Ser 727) in the nuclei compared with CNE1. Stronger signals were observed for $\mathrm{pY}$-stat 3 than for $\mathrm{pS}$-stat3 providing important evidence that under LMP1 regulation phosphorylated STAT3 (particularly pY-stat3) had nuclear accumulation in NPC tissues. The data suggest that phosphorylated STAT3 (Tyr 705, Ser 727) nuclear translocation may be Tyr 705-phosphorylation dependent in LMP1-positive cells (Fig. 5).

JAK mediates LMP1-induced STAT3 Tyr 705 phosphorylation. Upon confirming the promotion of STAT3 (Tyr 705, Ser 727) phosphorylation and nuclear translocation by LMP1, we further investigated the mechanism of LMP1 regulation of STAT3. Our previous study suggested that JAK3 activity was involved in LMP1 activation of STAT3, which required the cooperation of the CTAR2 and CTAR3 domains (28). As shown in Fig. 3, treating CNE1-LMP1 cells with WHI-P131 (31-33), a JAK3 inhibitor, for $24 \mathrm{~h}$ effectively inhibited JAK3-induced STAT3 Tyr 705 phosphorylation. In response to the inhibition, STAT3 Tyr 705 phosphorylation markedly decreased, but STAT3 Ser 727 phosphorylation and STAT3 protein levels did not change (Fig. 6). Thus, JAK3 was particularly important to STAT3 Tyr 705 phosphorylation induced by LMP1.

ERK mediates LMP1-induced STAT3 Ser 727 phosphorylation. To determine the role of ERK in LMP1-induced STAT3 Ser 727 phosphorylation, the activity of ERK was analyzed in the presence of the specific inhibitor PD98059 in CNE1-LMP1 cells and compared with control CNE1 cells. ERK was immunoprecipitated, and its activity was determined by kinase

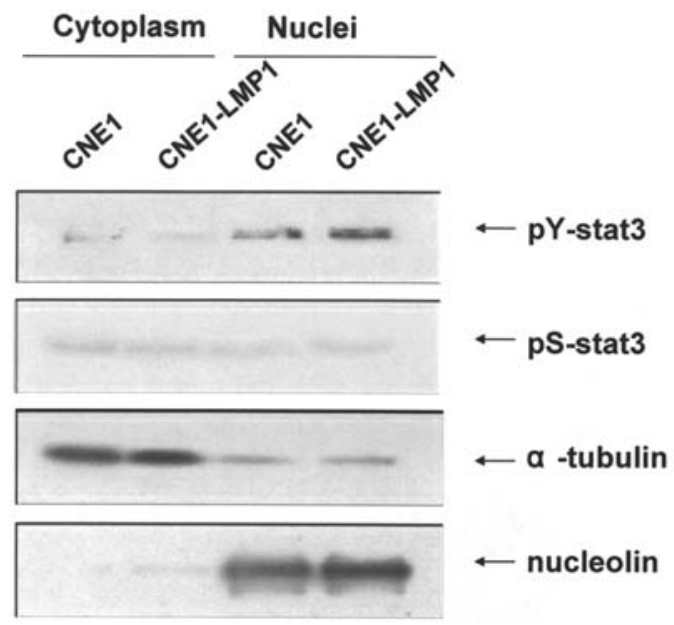

Figure 4. Nuclear translocation of phospho-STAT3 induced by LMP1 Cytoplasmic and nuclear proteins of CNE1 and CNE1-LMP1 cells were prepared, and Western blot analysis was performed. pY-stat3, pS-stat3, $\alpha$-tubulin, and nucleolin were examined. Nucleolin and $\alpha$-tubulin were the controls for nuclear protein and cytoplasmic protein, respectively. LMP1 promoted pY-stat 3 and pS-stat 3 nuclear translocation.

assays in vitro using Elk as a substrate. In comparison to CNE1, the CNE1-LMP1 cells showed an increase in ERK activity (Fig. 7A). The ERK activation was suppressed by PD98059 in a dose-dependent manner in CNE1-LMP1 cells. To determine a correlation between ERK activation and the activation of STAT3 Ser 727 phosphorylation, we blocked the activation of ERKs using PD98059, the MEK1 inhibitor. As shown in Fig. 7B, treatment with PD98059 caused a decrease in the ERK phosphorylation induced by LMP1. The levels of phosphorylated STAT3 Ser 727 in CNE1-LMP1 cells were reduced in a dose-dependent manner by PD98059. Total cellular levels of STAT3 ERK and STAT3 Tyr 705 phosphorylation, however, remained unchanged (Fig. 7B). These results strongly suggest that ERK is responsible for LMP1-induced STAT3 Ser 727 phosphorylation.

It was previously reported that JAK3 activation occurred upon ligand-mediated receptor multimerization and that the activated JAK3 subsequently phosphorylated STAT3 at a conserved tyrosine residue. This phosphotyrosine permitted the STAT3 to form dimers and enter the nuclei. To determine whether LMP1-mediated nuclear localization of STAT3 is an event dependent on the phosphorylation of STAT3 on tyrosines and/or serines, nuclear translocation of phosphorylated STAT3 (Tyr 705, Ser 727) was determined by laser scanning confocal microscopy with anti-FITC antibodies and rhodamineconjugated secondary antibody. STAT3 phosphorylation and translocation into the nucleus decreased after being in the presence of WHI-P131 for $24 \mathrm{~h}$ in CNE1-LMP1 cells. As shown in Fig. 8, we did not observe the same decline in nuclear accumulation of phosphorylated STAT3 (Tyr 705, Ser 727) when CNE1-LMP1 cells were treated with PD98059, a smallmolecule MEK1 inhibitor, for $2 \mathrm{~h}(34,35)$. The data suggested that phosphorylated STAT3 (Tyr 705, Ser 727) nuclear translocation was Tyr 705 phosphorylation dependent and Ser 727 phosphorylation independent.

A close association of phosphorylated STAT3 with NPC stage. To evaluate the possible effects of STAT3 phos- 


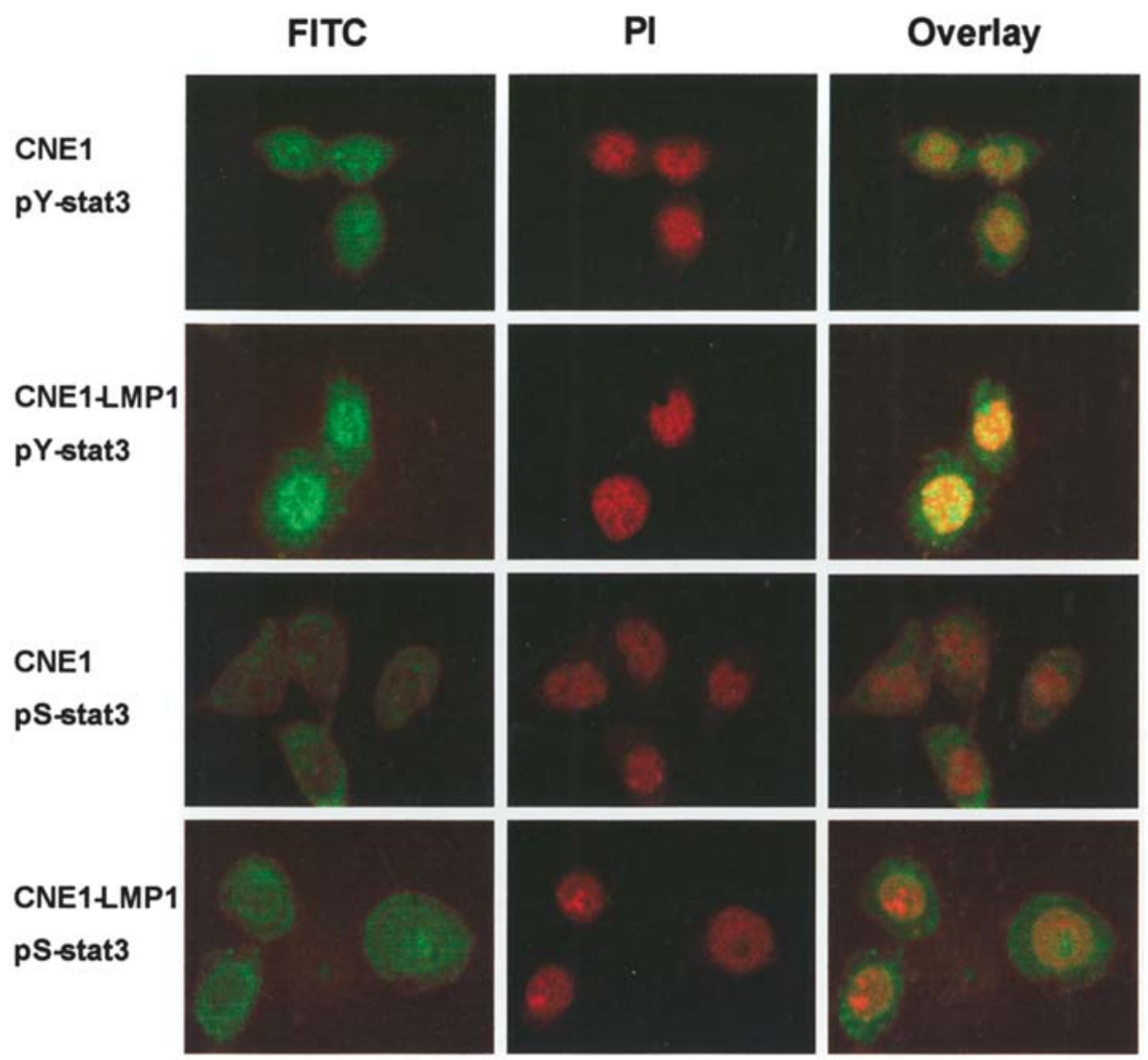

Figure 5. Fluorescence microscopy shows nuclear translocation of pY-stat3 and pS-stat3 induced by LMP1. FITC-labeled anti-rabbit IgG is specific for pSTAT3. Cells were counterstained with propidium iodide to display the nuclei. The fluorescent images were visualized with a laser scanning confocal microscope $(x 1,000)$. When the two images were merged, yellow signals were observed indicating the localization of phospho-STAT3 in nuclei. PBS was substituted for antibody as a blank control.

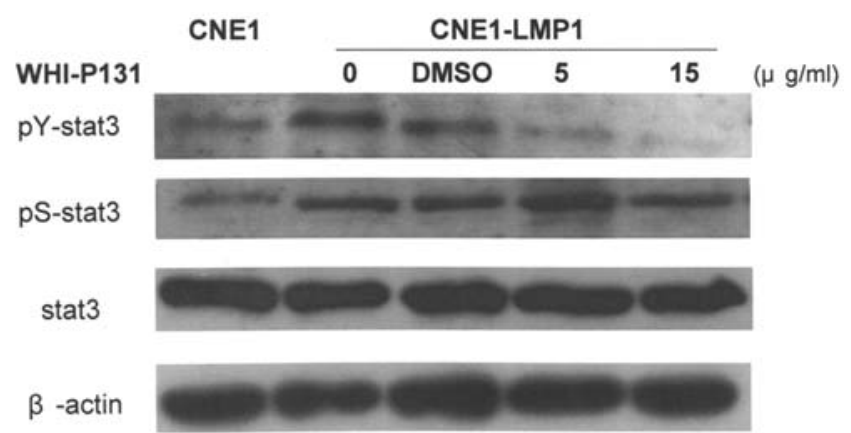

Figure 6. JAK3 mediates LMP1-induced STAT3 Tyr 705 phosphorylation. WHI-P131 $(10 \mu \mathrm{g} / \mathrm{ml})$ inhibited JAK3-induced tyrosine phosphorylation of STAT3 in CNE1-LMP1 cells. CNE1-LMP1 cells were treated with WHIP131, a JAK3 inhibitor, for $24 \mathrm{~h}$. Cell lysates were prepared, and equal amounts of protein were separated by $10 \%$ SDS-PAGE and blotted onto a nitrocellulose membrane. Quantification of immunoband intensities was determined by densitometry scanning, with B-actin as an internal control. LMP1 increased STAT3 tyrosine 705 phosphorylation through JAK3.

phorylation and LMP1 expression on the progression of NPC, immunohistochemisty (IHC) was performed on formalinfixed, paraffin-embedded tissue sections of human NPC using the EnVision Doublestain system to determine the expression of phosphorylated STAT3 and LMP1. Coexpression of phosphorylated STAT3 and LMP1 signals were distinctly observed, and pY-stat 3 and pS-stat 3 were mainly located in
Table II. Relativity of LMP1 and pY-stat3, pS-stat3 in 40 NPC tissue samples.

\begin{tabular}{lccc}
\hline Group & $\mathrm{n}$ & $\mathrm{pY}$-stat3 & pS-stat3 \\
\hline LMP1 (-) & 15 & $(7) 46.7 \%$ & $(6) 40.0 \%$ \\
LMP1 (+) & 25 & $(20) 80.0 \%$ & $(18) 72.0 \%$ \\
$\mathrm{r}_{\mathrm{s}}$ & & 0.345 & 0.316 \\
$\mathrm{p}$ & & 0.029 & 0.047 \\
\hline
\end{tabular}

Statistical analysis of the correlation of LMP1 and pY-stat3 or pSstat3 in 40 tissue samples. from NPC patients. The EnVision Doublestain method was applied as described. Data show that LMP1 correlates with both pY-stat $3\left(\mathrm{r}_{\mathrm{s}}=0.345, \mathrm{p}=0.029\right)$ and $\mathrm{pS}$ stat3 $\left(r_{\mathrm{s}}=0.316, \mathrm{p}=0.047\right)$.

the nuclei (Fig. 9). The IHC Doublestain technique was also applied to 40 NPC patient tissue samples to evaluate the relationship between LMP1 and phosphorylated STAT3 (Tyr 705, Ser 727), as well as the correlation of phosphorylated STAT3 (Tyr 705, Ser 727) with NPC clinical stages. Statistical analysis indicated that the positive percentage of LMP1 in NPC patient tissues was $\sim 62.5 \%(25 / 40)$, the positive percentage of pY-stat3 was $\sim 67.5 \%$ (27/40), and the positive percentage of $\mathrm{pS}$-stat3 was $\sim 60.0 \%$ (24/40). 
A

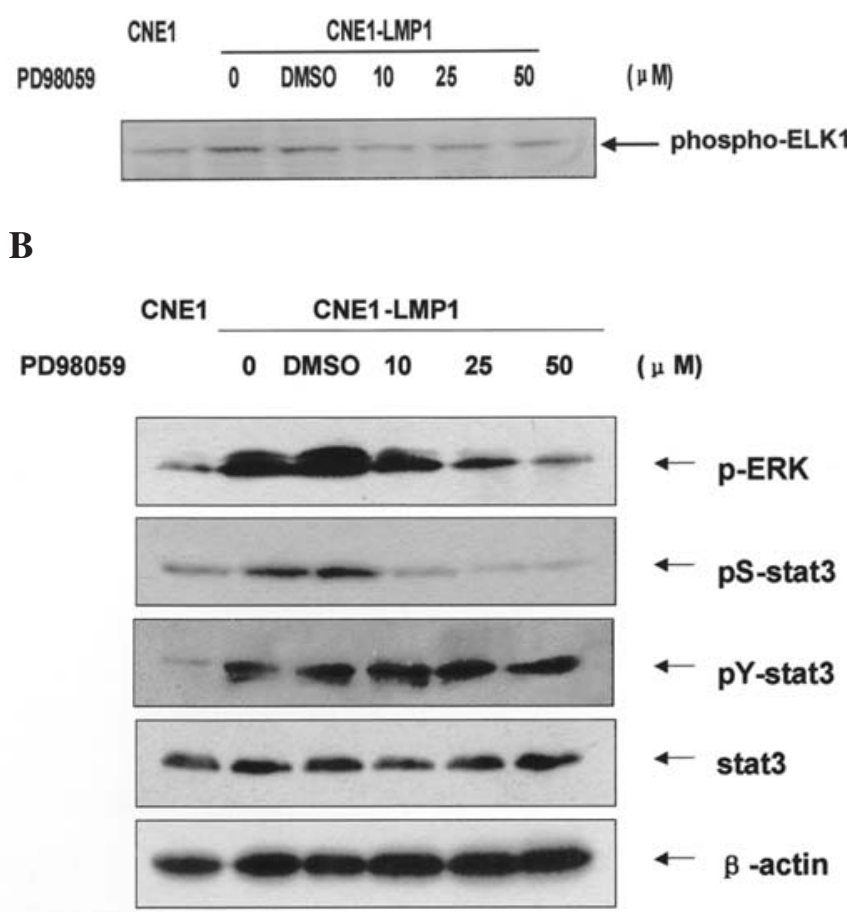

Figure 7. (A) LMP1 induces the activity of ERK. Phosphorylation inhibitor PD98059 inhibited p44/42 MAPK activity in CNE1-LMP1 cells. CNE1 cells were used as a negative control. (B) ERK mediates LMP1-induced STAT3 Ser 727 phosphorylation. ERK phosphorylation inhibitor PD98059 inhibited serine phosphorylation of STAT3 in CNE1-LMP1 cells. CNE1LMP1 cells were treated with PD98059 for $2 \mathrm{~h}$. Cell lysates were prepared, and equal amounts of protein were separated by $10 \%$ SDS-PAGE and blotted onto a nitrocellulose membrane. Quantification of immunoband intensities was determined by densitometry scanning, with $\beta$-actin as an internal control.

Moreover, the positive percentage of pY-stat3 was $~ 80.0 \%$ (20/25) in LMP1-positive tissue and 46.7\% (7/25) in LMP1negative tissue $\left(\mathrm{r}_{\mathrm{s}}=0.345, \mathrm{p}=0.029\right)$. The positive percentage of pS-stat3 was $72.0 \%$ (18/25) in LMP1-positive tissue and $40.0 \%(6 / 15)$ in LMP1-negative tissue $\left(\mathrm{r}_{\mathrm{s}}=0.316, \mathrm{p}=0.047\right)$ (Table II). Thus, there existed a marked statistical difference. Of the 40 NPC patient tissue samples, 8 were CIN I or II, and 32 were CIN III or IV. Of the 8 that were CIN I or II, $25.0 \%$ $(2 / 8)$ had a positive proportion of pY-stat3, and $62.5 \%(5 / 8)$ had a positive proportion of $\mathrm{pS}$-stat 3 . Of the 32 that were CIN III or IV, $78.1 \%(25 / 32)$ had a positive proportion of pY-stat3, and 59.4\% (19/32) had a positive proportion of pSstat3. These results revealed the relevancy of LMP1 with phospho-STAT3 after statistic analysis $(\mathrm{P}<0.05)$ and a close association of phosphorylated STAT3 (Tyr 705, Ser 727) with the development of NPC (Table III).

\section{Discussion}

EBV-LMP1 is one of several latently expressed genes essential for the growth transformation of B lymphocytes in vitro (3). The frequent expression of LMP1 in undifferentiated NPC points to a role for this viral oncoprotein as a key effector molecule in NPC pathogenesis. LMP1 affects the control of cellular growth and differentiation of epithelial cells in vitro. The underlying molecular events behind this are still unknown.
Table III. Relativity of pY-stat3, pS-stat3 and clinical stages in 40 NPC tissue samples.

\begin{tabular}{lccc}
\hline Clinical stages & $\mathrm{n}$ & $\mathrm{pY}$-stat3 & $\mathrm{pS}$-stat3 \\
\hline I, II & 8 & (2) $25.0 \%$ & (5) $62.5 \%$ \\
III, IV & 32 & (25) $78.1 \%$ & (19) $59.4 \%$ \\
$\quad \chi^{2}$ & & 4.167 & 0.000 \\
$\mathrm{p}$ & & 0.041 & 1.000 \\
\hline
\end{tabular}

Statistical analysis of the correlation of pSTAT3 with NPC clinical stages in 40 tissue samples from NPC patients. The EnVision Doublestain method was applied as described. Data show that pYstat3, but not $\mathrm{pS}$-stat3, correlates with clinical stages III and IV.

The goals of this study were to demonstrate that LMP1 is capable of activating STAT3 through a complex mechanism involving the MAPK/ERK and JAK/STAT pathways that directly phosphorylate STAT3 at Ser 727 and Tyr 705, respectively. LMP1 resulted in increased phosphorylation of STAT3 at Tyr 705 and Ser 727 in CNE1-LMP1 cells as compared with CNE1 cells. Blocking of extracellular IL-6 with anti-IL-6 neutralizing antibody abolished the LMP1-mediated increase in STAT3 tyrosine and serine phosphorylation in Tet-on-LMP1-HNE2 cells. The tyrosine phosphorylation of STAT3 induced by LMP1 led to functional activation, as demonstrated by the ability of LMP1 to induce nuclear translocation of STAT3 in CNE1-LMP1 cells.

LMP1 is known to engage signaling on the JAK/STAT pathway, and inhibition of this pathway by coexpression of a constitutively active CTAR2 mutant abolished STAT3 pTKs3 luciferase reporter activity (28). Mutational analysis of the LMP1 cytoplasmic $\mathrm{COOH}$ terminus identified both the CTAR2 and CTAR3 domains as contributing to JAK/STAT signaling. The effects of JAK on LMP1-mediated STAT3 activation were found to occur as treatment of CNE1-LMP1 cells with WHI-P131 partially inhibited the upregulation of STAT3 Tyr 705 phosphorylation. This suggests that LMP1 modulates the IL-6 and JAK pathways that phosphorylate STAT3 Tyr 705 indirectly or directly, respectively.

In addition to tyrosine phosphorylation, we found that STAT3 is serine phosphorylated at a single residue (Ser 727) in response to IL-6. At present, the signaling events downstream of the gp130 receptor have not been fully explored, and the kinase responsible for IL-6-induced STAT3 Ser 727 phosphorylation has not been identified. STAT3 Ser 727 is phosphorylated via different pathways under different stimulations. Receptor tyrosine kinases, IL-2, the B cell receptor $(\mathrm{BCR})$, and the $\mathrm{T}$ cell receptor (TCR) phosphorylate STAT3 Ser 727 via the MAPK/ERK pathway $(36,37)$; ultraviolet (UV) radiation, high saturation stress, tumor necrosis factor (TNF)- $\alpha$, and lipopolysaccharide (LPS) induce STAT3 Ser 727 phosphorylation via the MAPK/Jun N-terminal kinase (JNK) pathway; v-src stimulates STAT3 Ser 727 phosphorylation via MAPK/p38 (38); and IL-6 activates STAT3 Ser 727 phosphorylation through the protein kinase C (PKC) pathway (39). The binding of IL-6 to its cellular receptor simultaneously activates both ERK1/2 and STAT3 $(40,41)$. 


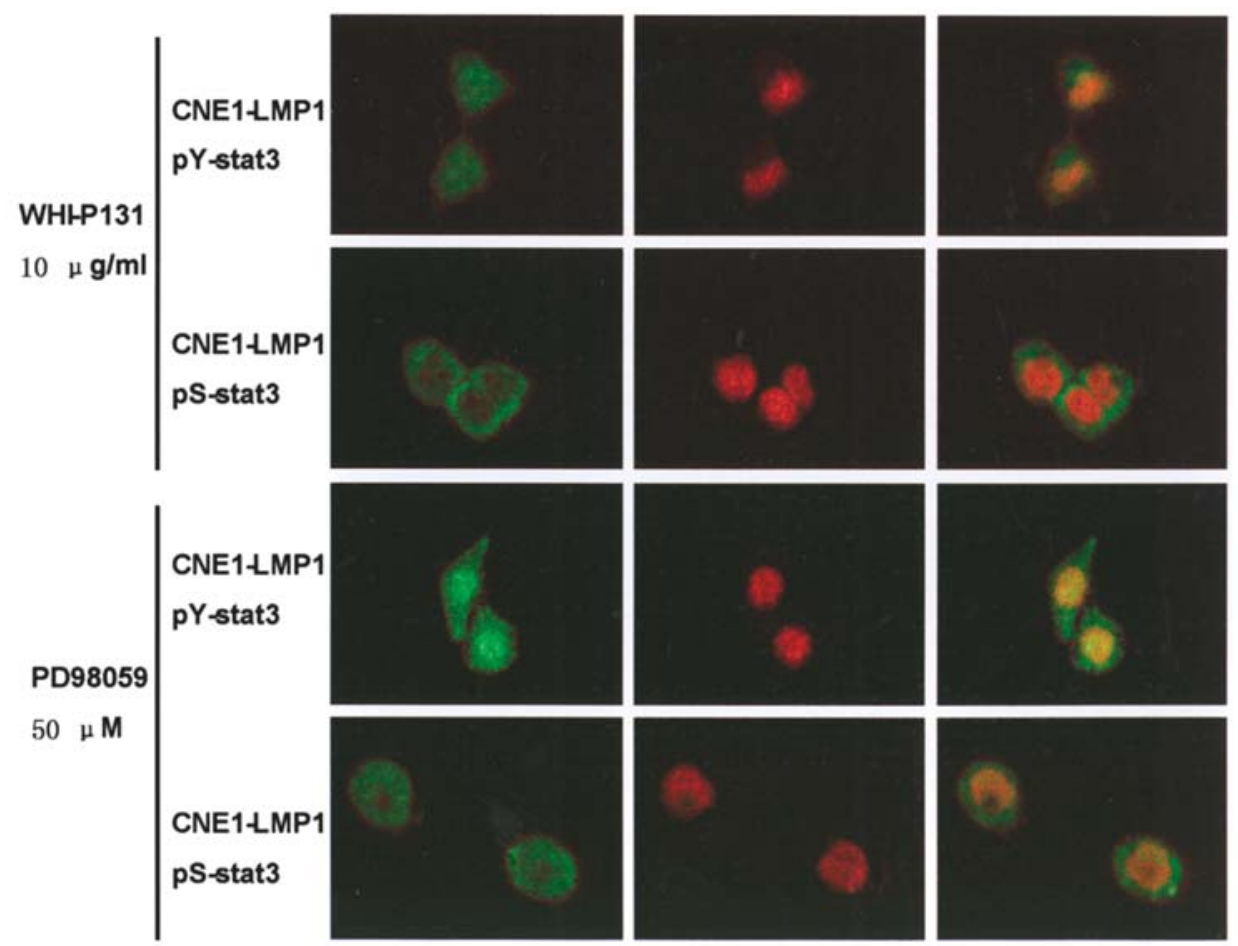

Figure 8. Fluorescence microscopy shows nuclear translocation of pY-STAT3 and pS-STAT3 induced by LMP1 in a tyrosine 705 phosphorylated STAT3dependent manner. FITC-labeled anti-rabbit IgG is specific for pSTAT3. Cells were counterstained with propidium iodide to display the nuclei. The fluorescent images were visualized with a laser scanning confocal microscope $(\mathrm{x} 1,000)$. When the two images were merged, yellow signals were observed indicating the localization of phospho-STAT3 in nuclei. PBS was substituted for antibody as a blank control. CNE1-LMP1 cells were treated with WHI-P131 $(10 \mu \mathrm{g} / \mathrm{ml})$ for $24 \mathrm{~h}$ and with PD98059 $(50 \mu \mathrm{M})$ for $2 \mathrm{~h}$. Data showed that LMP1 induced the nuclear localization of phospho-STAT3 in a tyrosine $705-$ phosphorylated STAT3-dependent manner.

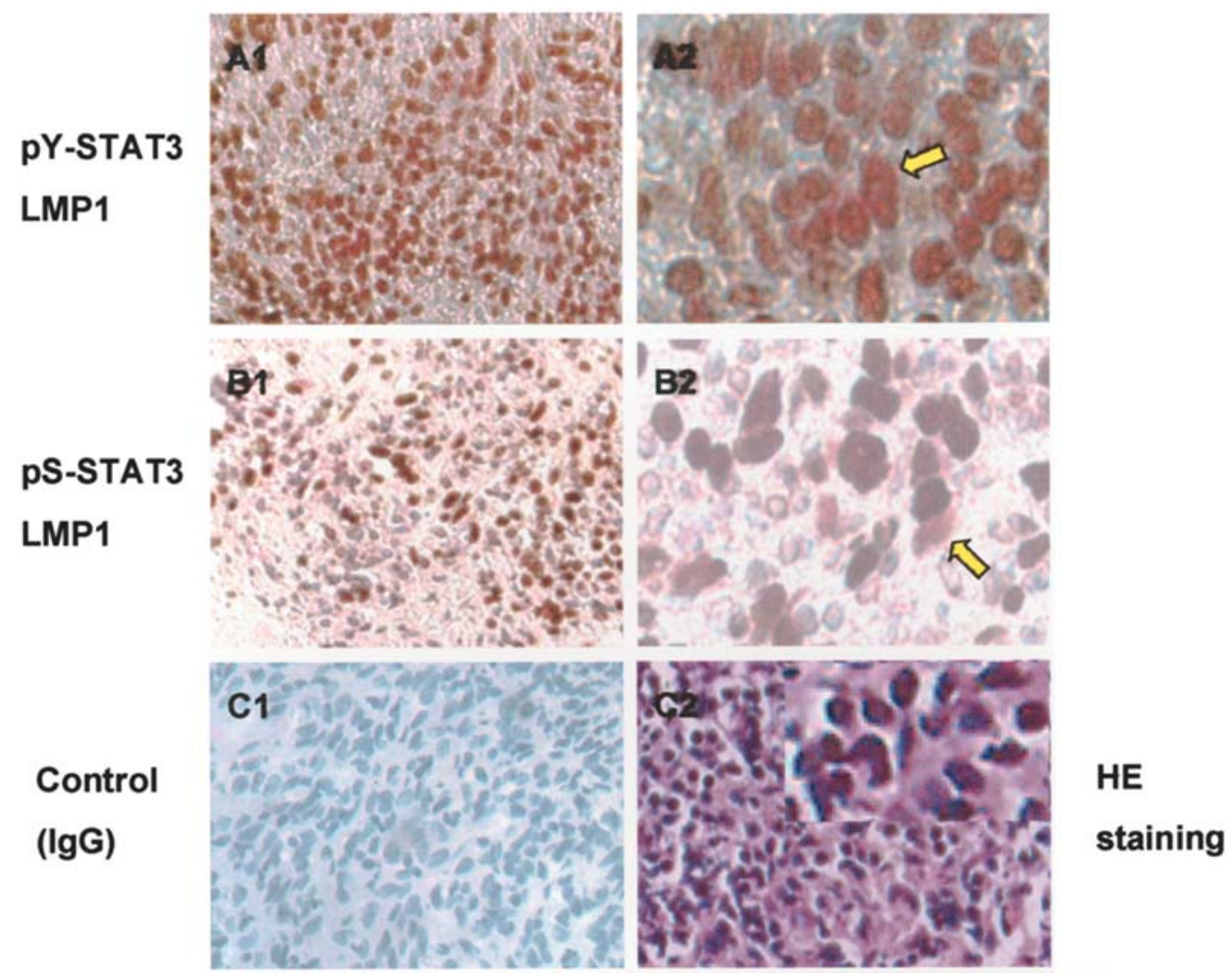

Figure 9. Coexpression of pY-STAT3 or pS-STAT3 with LMP1 in NPC tissue with the IHC EnVision Doublestain method. (A1) pY-stat3 (brown) and LMP1 (red), x150; (A2) pY-stst3 (brown) and LMP1 (red), x300; (B1) pS-stat3 (brown) and LMP1 (red), x150; (B2) pS-stat3 (brown) and LMP1 (red), x300; (C1) control, x300; (C2) hematoxylin and eosin (HE) staining, x150 (top right corner, x300). Control, mouse and rabbit IgG. Cells were counterstained with hematoxylin to display the nuclei. Mouse and rabbit IgG was substituted for the antibody as a blank control. Data show that LMP1 induced the nuclear localization of phospho-STAT3 in NPC tissues. 
In this study, we provide evidence of another signaling pathway activated by LMP1 that induces STAT3 Ser 727 phosphorylation. We showed that LMP1 expression led to the activation of ERK, as evidenced by the ability of LMP1 to induce ERK phosphorylation. Furthermore, ERK immunoprecipitated from LMP1-positive cells induced the phosphorylation of Elk, one of the downstream targets of ERK. Since LMP1-induced IL-6 signaling engages STAT3 Ser 727 phosphorylation, the question of whether these two pathways run on the same axis or in parallel has not been clarified. We also demonstrated that PD98059 inhibited ERK activity and phosphorylation in CNE1-LMP1 cells. Thus, the functional implications of ERK-mediated STAT3 Ser 727 phosphorylation were demonstrated by the ability of PD98059 to downregulate LMP1-activated STAT3 Ser 727 phosphorylation. The JNK pathway did not influence STAT3 phosphorylation, which eliminates the possibility of JNK involvement in the process of LMP1-induced STAT3 Ser 727 phosphorylation (data not shown). Furthermore, LMP1 promotion of phosphorylated STAT3 nuclear translocation has been observed. This process is STAT3 Tyr 705-phosphorylation dependent and Ser 727-phosphorylation independent.

pY-stat3 has a high correlation with NPC stages III and IV, suggesting its role in NPC invasion and metastasis by IHC Doublestain. We conclude that the overexpression of $\mathrm{p}$ STAT3 may have an important role in the development of NPC tumors. Abnormal activation of STAT3 may be related to the metastasis potential in NPC, and the simultaneous detection of p-stat 3 and LMP1 may aid in predicting the progression of NPC. STAT3 promotes metastasis and angiogenesis by inducing expression of the metastasis gene matrix metalloproteinase-2 (MMP-2) and the potent angiogenic gene VEGF $(20,22)$. Our present findings contribute toward expanding the knowledge of the mechanism of NPC metastasis and in identifying new predictive biomarkers and therapeutic targets.

Overall, this study demonstrates the ability of a viral protein, EBV-encoded LMP1, to activate the MAPK/ERK and JAK/STAT pathways responsible for LMP1-induced STAT3 activation. The continued discovery of unique signaling pathways and the identification of crucial signaling molecules greatly enhances the current knowledge of intracellular mechanisms involved in LMP1 signaling.

\section{Acknowledgements}

This research is supported by a National Nature Science Grant (no. 30572111) and the Development Projects of National Critical Basic Research entitled 'Fundamental studies on carcinogenesis and prevention and therapy for human cancers' (no. 2004CB518703) and 'The mechanism of invasion and metastasis and the molecular obstruction of metastasis' (no. 2002CB513100).

\section{References}

1. Rickinson AB: Epstein-Barr virus in action in vivo. $\mathrm{N}$ Engl $\mathrm{J}$ Med 338: 1461-1463, 1998.

2. Fahraeus R, Rymo L, Rhim JS and Klein G: Morphological transformation of human keratinocytes expressing the LMP gene of Epstein-Barr virus. Nature 345: 447-449, 1990.
3. Peng $M$ and Lundgren E: Transient expression of the EpsteinBarr virus LMP1 gene in human primary B cells induces cellular activation and DNA synthesis. Oncogene 7: 1775-1782, 1992.

4. Eliopoulos AG and Young LS: LMP1 structure and signal transduction. Semin Cancer Biol 11: 435-444, 2001.

5. Hennessy K, Fennewald S, Hummel M, Cole T and Kieff E: A membrane protein encoded by Epstein-Barr virus in latent growth-transforming infection. Proc Natl Acad Sci USA 81: 7207-7211, 1984

6. Izumi KM and Kieff ED: The Epstein-Barr virus oncogene product latent membrane protein 1 engages the tumor necrosis factor receptor-associated death domain protein to mediate B lymphocyte growth transformation and activate NF-kappaB. Proc Natl Acad Sci USA 94: 12592-12597, 1997.

7. Huen DS, Henderson SA, Croom-Carter D and Rowe M: The Epstein-Barr virus latent membrane protein-1 (LMP1) mediates activation of NF-kappa B and cell surface phenotype via two effector regions in its carboxy-terminal cytoplasmic domain. Oncogene 10: 549-560, 1995.

8. Gires O, Kohlhuber F, Kilger E, Baumann M, Kieser A, Kaiser C, Zeidler R, Scheffer B, Ueffing M and Hammerschmidt W: Latent membrane protein 1 of Epstein-Barr virus interacts with JAK3 and activates STAT proteins. EMBO J 18: 3064-3073, 1999

9. Fanger GR, Gerwins P, Widmann C, Jarpe MB and Johnson GL: MEKKs, GCKs, MLKs, PAKs, TAKs, and tpls: upstream regulators of the c-Jun amino-terminal kinases? Curr Opin Genet Dev 7: 67-74, 1997.

10. Lim L, Manser E, Leung T and Hall C: Regulation of phosphorylation pathways by $\mathrm{p} 21$ GTPases. The p21 Ras-related Rho subfamily and its role in phosphorylation signalling pathways. Eur J Biochem 242: 171-185, 1996.

11. Symons M: Rho family GTPases: the cytoskeleton and beyond. Trends Biochem Sci 21: 178-181, 1996.

12. Hu Z, Zeng L, Tao YG, Tang FQ, Wang H, Luo FJ, Yi W and Cao Y: EB virus-encoded latent membrane protein 1 activates the JNK signalling pathway via a mechanism involving TRADD and TRAF in nasopharyngeal carcinoma cell. Prog Biochem Biophys 29: 562-566, 2002.

13. Zeng L, Li M, Song X, Tao YG, Tang M, Li W and Cao Y: Epstein-barr virus latent membrane protein 1 (LMP1) activated Ets-1 expression mediated by ERK in nasopharyngeal cell line. Prog Biochem Biophys 30: 574-578, 2003.

14. Yang J, Chatterjee-Kishore M, Staugaitis SM, Nguyen H, Schlessinger K, Levy DE and Stark GR: Novel roles of unphosphorylated STAT3 in oncogenesis and transcriptional regulation. Cancer Res 65: 939-947, 2005.

15. Bienvenu F, Barre B, Giraud S, Avril S and Coqueret O: Transcriptional regulation by a DNA-associated form of cyclin D1. Mol Biol Cell 16: 1850-1858, 2005.

16. Kanda N, Seno H, Konda Y, Marusawa H, Kanai M, Nakajima T, Kawashima T, Nanakin A, Sawabu T, Uenoyama Y, et al: STAT3 is constitutively activated and supports cell survival in association with survivin expression in gastric cancer cells. Oncogene 23: 4921-4929, 2004.

17. Wang T, Niu G, Kortylewski M, Burdelya L, Shain K, Zhang S, Bhattacharya R, Gabrilovich D, Heller R, Coppola D, et al: Regulation of the innate and adaptive immune responses by Stat-3 signaling in tumor cells. Nat Med 10: 48-54, 2004.

18. Cheng F, Wang HW, Cuenca A, Huang M, Ghansah T, Brayer J, Kerr WG, Takeda K, Akira S, Schoenberger SP, et al: A critical role for Stat3 signaling in immune tolerance. Immunity 19: 425-436, 2003.

19. Vultur A, Cao J, Arulanandam R, Turkson J, Jove R, Greer P, Craig A, Elliott B and Raptis L: Cell-to-cell adhesion modulates Stat 3 activity in normal and breast carcinoma cells. Oncogene 23: 2600-2616, 2004

20. Hua Yu RJ: The STATs of cancer - new molecular targets come of age. Nat Rev Cancer 4: 97-105, 2004.

21. Rivat C, De Wever O, Bruyneel E, Mareel M, Gespach C and Attoub S: Disruption of STAT3 signaling leads to tumor cell invasion through alterations of homotypic cell-cell adhesion complexes. Oncogene 23: 3317-3327, 2004.

22. Xie TX, Wei D, Liu M, Gao AC, Ali-Osman F, Sawaya R and Huang S: Stat3 activation regulates the expression of matrix metalloproteinase-2 and tumor invasion and metastasis. Oncogene 23: 3550-3560, 2004.

23. Chen H, Lee JM, Zong Y, Borowitz M, Ng MH, Ambinder RF and Hayward SD: Linkage between STAT regulation and Epstein-Barr virus gene expression in tumors. J Virol 75 : 2929-2937, 2001. 
24. Schuringa JJ, Schepers H, Vellenga E and Kruijer W: Ser727dependent transcriptional activation by association of p300 with STAT3 upon IL-6 stimulation. FEBS Lett 495: 71-76, 2001.

25. Turkson J and Jove R: STAT proteins: novel molecular targets for cancer drug discovery. Oncogene 19: 6613-6626, 2000.

26. O'Rourke L and Shepherd PR: Biphasic regulation of extracellular-signal-regulated protein kinase by leptin in macrophages: role in regulating STAT3 Ser727 phosphorylation and DNA binding. Biochem J 364: 875-879, 2002.

27. Chen H, Hutt-Fletcher L, Cao L and Hayward SD: A positive autoregulatory loop of LMP1 expression and STAT activation in epithelial cells latently infected with Epstein-Barr virus. J Virol 77: 4139-4148, 2003.

28. Tan YN, Tao YG, Li LL and Cao Y: Stat3 pathway is activated by EB virus encoded latent membrane protein 1 in nasopharyngeal carcinoma cells. EBV Symposium 148, 2004.

29. Tan YN, Tao YG, Li LL, Liu SF, Tang M, Gu HH and Cao Y: Regulation of human VEGF by expression latent membrane protein 1 encoded by EB virus via STAT3 in nasopharyngeal carcinoma cell line. Prog Biochem Biophys 31: 427, 2004.

30. Liao W, Yi H, Li XY, Tang M, Gu HH and Cao Y: Doxycyclinedependent expression of Epstein-Barr virus latent membrane protein 1 with Tet regulating system in nasopharygeal carcinoma cell line. Sheng Wu Hua Xue Yu Sheng Wu Wu Li Xue Bao 31: 309-312, 1999.

31. Barata JT, Boussiotis VA, Yunes JA, Ferrando AA, Moreau LA, Veiga JP, Sallan SE, Look AT, Nadler LM and Cardoso AA: IL-7-dependent human leukemia T-cell line as a valuable tool for drug discovery in T-ALL. Blood 103: 1891-1900, 2004.

32. Sudbeck EA, Liu XP, Narla RK, Mahajan S, Ghosh S, Mao C and Uckun FM: Structure-based design of specific inhibitors of Janus kinase 3 as apoptosis-inducing antileukemic agents. Clin Cancer Res 5: 1569-1582, 1999.
33. Tibbles HE, Vassilev A, Wendorf H, Schonhoff D, Zhu D, Lorenz D, Waurzyniak B, Liu XP and Uckun FM: Role of a JAK3-dependent biochemical signaling pathway in platelet activation and aggregation. J Biol Chem 276: 17815-17822, 2001.

34. Alessi DR, Cuenda A, Cohen P, Dudley DT and Saltiel AR: PD 098059 is a specific inhibitor of the activation of mitogenactivated protein kinase kinase in vitro and in vivo. J Biol Chem 270: 27489-27494, 1995.

35. Tanaka K AM and Sato Y: Roles of extracellular signalregulated kinase $1 / 2$ and p38 mitogen-activated protein in the signal transduction of basic fibroblast growth factor in endothelial cells during angiogenesis. Jpn J Cancer Res 90: 647-654, 1999.

36. $\mathrm{Ng} \mathrm{J}$ and Cantrell D: STAT3 is a serine kinase target in T lymphocytes. Interleukin 2 and $\mathrm{T}$ cell antigen receptor signals converge upon serine 727. J Biol Chem 272: 24542-24549, 1997.

37. Chung J, Uchida E, Grammer TC and Blenis J: STAT3 serine phosphorylation by ERK-dependent and -independent pathways negatively modulates its tyrosine phosphorylation. Mol Cell Biol 17: 6508-6516, 1997.

38. Turkson J, Bowman T, Adnane J, Zhang Y, Djeu JY, Sekharam M, Frank DA, Holzman LB, Wu J, Sebti S and Jove R: Requirement for Ras/Rac1-mediated p38 and c-Jun N-terminal kinase signaling in Stat3 transcriptional activity induced by the Src oncoprotein. Mol Cell Biol 19: 7519-7528, 1999.

39. Jain N, Zhang T, Kee WH, Li W and Cao X: Protein kinase C delta associates with and phosphorylates Stat 3 in an interleukin6-dependent manner. J Biol Chem 274: 24392-24400, 1999.

40. Taga T and Kishimoto T: Gp130 and the interleukin- 6 family of cytokines. Annu Rev Immunol 15: 797-819, 1997.

41. Kishimoto T, Taga T and Akira S: Cytokine signal transduction. Cell 76: 253-262, 1994. 